\author{
Corpus \\ $14 \mid 2015$ \\ Constitution et usage de corpus en linguistique \\ berbère
}

\title{
Le syntagme prépositionnel à la périphérie gauche en Taqbaylit
}

Sabrina Bendjaballah, Samir Ben Si Saïd and Martin Haiden

\section{OpenEdition \\ Journals}

Electronic version

URL: http://journals.openedition.org/corpus/2710

ISSN: 1765-3126

\section{Publisher}

Bases; corpus et langage - UMR 6039

\section{Printed version}

Date of publication: 1 January 2015

Number of pages: $263-280$

ISBN: 1 638-9808

ISSN: 1638-9808

\section{Electronic reference}

Sabrina Bendjaballah, Samir Ben Si Saïd and Martin Haiden, « Le syntagme prépositionnel à la périphérie gauche en Taqbaylit», Corpus [Online], 14 | 2015, Online since 29 August 2017, connection on 08 September 2020. URL : http://journals.openedition.org/corpus/2710

This text was automatically generated on 8 September 2020

(C) Tous droits réservés 


\title{
Le syntagme prépositionnel à la périphérie gauche en Taqbaylit
}

\author{
Sabrina Bendjaballah, Samir Ben Si Saïd and Martin Haiden
}

\section{Le problème : la position de la préposition dans les constructions interrogatives prépositionnelles}

Dans les langues berbères, l'interrogation sur un groupe prépositionnel déclenche l'emploi de constructions particulières (pour des informations pertinentes, on pourra consulter, entre de nombreux autres, Penchoen 1973, Chaker 1983, Guerssel 1987, Bendjaballah \& Haiden 2005, 2013a, b, Mettouchi 2005, Ouali 2011, Ouhalla \& El Hankari 2012/ce volume). Dans cet article, nous analysons ces constructions sur la base de données recueillies dans un réseau de 10 points d'enquête en Kabylie. L'analyse syntaxique, pensons-nous, ne peut reposer que sur la segmentation et l'identification précise de la distribution des éléments impliqués dans les structures examinées. Ceci suppose de prendre au sérieux la dimension morpho-phonologique de ces structures. Cet article fait ainsi partie d'un projet plus large portant sur la phonologie, la morphologie et la syntaxe des groupes prépositionnels dans différentes variétés du berbère kabyle (détails en section 2).

Dans le parler de Chemini (Petite Kabylie), les questions portant sur un groupe prépositionnel sont caractérisées par la préfixation de la préposition sur le complémenteur et la réalisation du complément nominal de la préposition à la gauche du complexe préposition-complémenteur (Bendjaballah \& Haiden 2005, 2013a, b). Ainsi, dans les configurations (1a, b), le complémenteur (c) est morphologiquement complexe: il est composé de la base $\mathrm{C}$ et du préfixe $\mathrm{P}^{1}$. Ces configurations sont intéressantes parce qu'elles impliquent soit la séparation de la $\mathrm{P}$ de son complément nominal (1a), soit le redoublement de la P (1b). Notons qu'outre ( $1 \mathrm{a}, \mathrm{b})$, la configuration (1c) est également attestée à Chemini. Celle-ci nous intéressera cependant moins : elle 
correspond en effet à la configuration par défaut, que l'on retrouve dans de nombreuses langues, à savoir le déplacement d'un syntagme prépositionnel (PP) entier.

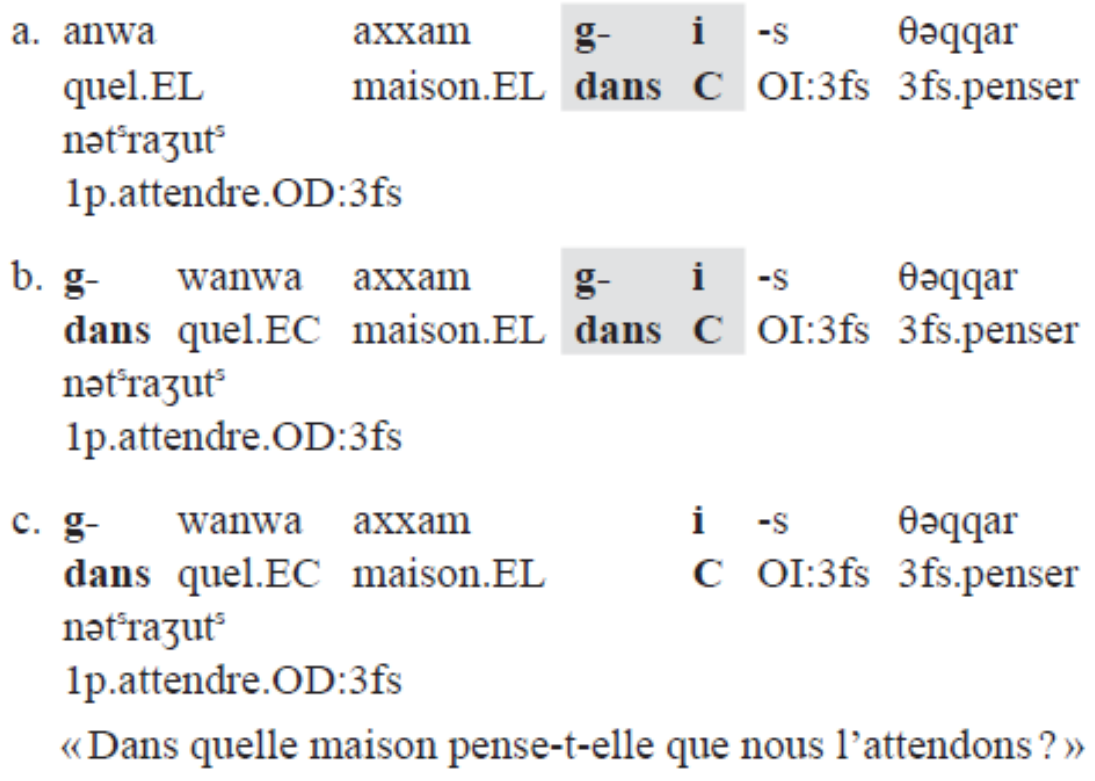

i $\quad$ s $\quad$ өəqqar

C OI:3fs 3 fs.penser

3 (1a) est problématique car un syntagme nominal (DP) ne peut pas directement prendre la fonction de complément prépositionnel. On pourrait envisager de proposer que le DP initial apparait séparé de sa P (cf. la structure anglaise " Where did he go to ?»). Cependant, une telle analyse implique que la $\mathrm{P}$ reste dans sa position d'origine. Or la $\mathrm{P}$ en (1a) apparaitt dans le domaine du complémenteur. De telles configurations ont certes été relevées, mais seulement dans des structures bien particulières, ce qui n'est pas le cas ici (voir par ex. le phénomène de sluicing, Merchant 2002). (1b) est problématique car la préposition est redoublée : en ce cas, l'hypothèse d'une séparation de DP et P est exclue. $^{2}$

4 Afin de rendre compte de ces constructions à complémenteur préfixé, Bendjaballah \& Haiden $(2005,2013 a, b)$ proposent une structure de prédication, représentée en (2) : le constituant interrogatif initial des structures ( $1 \mathrm{a}, \mathrm{b})$, anwa axxam / gwanwa axxam, ne fait pas formellement partie de la phrase, mais y est rattaché par une relation de prédication. Il se trouve dans une position externe à la phrase, que nous nommons ici «position (I) ». Etant externe à la phrase, ce constituant ne peut pas directement avoir la fonction grammaticale de complément prépositionnel. Cette fonction est remplie par un opérateur vide en position de spécificateur de C (position (II)), dont le contenu référentiel est déterminé par le constituant interrogatif initial. Le complémenteur (et son préfixe éventuel), enfin, est hébergé par la position (III).

\begin{tabular}{|l|l|l|l|l|}
\hline (2) & (I) & (II) & (III) & \\
\hline & $\begin{array}{l}\text { anwa axxam/ } \\
\text { gwanwa axxam }\end{array}$ & OP $_{\text {zéro }}$ & $g-i$ & $\ldots$ \\
\hline
\end{tabular}

Dans les constructions à complémenteur nu, i.e. non préfixé (type (1c)), l'hypothèse d'un opérateur vide n'est pas nécessaire, car le PP interrogatif initial peut directement 
prendre la fonction de complément prépositionnel. Il occupe donc la position (II), celle du spécificateur de C :

\begin{tabular}{|l|l|l|l|l|}
\hline$(3)$ & (I) & (II) & (III) & \\
\hline & & gwanwa axxam & $i$ & $\ldots$ \\
\hline
\end{tabular}

6 Dans cet article, nous partons de l'observation suivante. Notre corpus présente, comme à Chemini, (i) des complémenteurs morphologiquement complexes composés d'un préfixe prépositionnel et d'une base $\mathrm{C}$, (ii) des constituants interrogatifs, avec et sans $\mathrm{P}$, en tête de la phrase. En outre, et c'est le point crucial, un troisième type de structure est attesté, caractérisé par la présence d'un constituant entre le constituant interrogatif initial et le complémenteur complexe. Nous proposons que ce constituant est la manifestation morphologique de l'opérateur zéro représenté en (2) pour le parler de Chemini. En d'autres termes, la position (II), réalisée zéro à Chemini, fait surface dans divers parlers kabyles. Notre objectif est tout d'abord de décrire et analyser la réalisation morphologique de cette position(II) et ensuite de comprendre les conséquences sur le contenu morphologique de la position (III) du fait que cette position est réalisée.

7 Nos nouvelles données permettent ainsi de préciser les traits de l'opérateur zéro représenté en (2) ainsi que sa structure interne. Plus précisément, nous mettons au jour la généralisation suivante ${ }^{3}$ :

(4) Si, dans une construction interrogative portant sur un complément prépositionnel, le complémenteur (C) est spécifié par un constituant interrogatif, alors $\mathrm{C}$ ne porte pas de $\mathrm{P}$ préfixée. Si $\mathrm{C}$ est spécifié par un constituant non interrogatif ou bien si le spécificateur de $\mathrm{C}$ n'est pas réalisé morphologiquement, alors $\mathrm{C}$ est préfixé par $\mathrm{P}$.

\section{La constitution du corpus}

8 Nous sommes partis d'un corpus établi auprès d'une locutrice à L1 kabyle avec L2 français, native de Chemini en Kabylie orientale (Petite Kabylie, chef-lieu : Bejaïa, $c f$. Bendjaballah \& Haiden $(2005,2013 a, b)$ pour plus de détails), puis avons élargi le périmètre dialectal de la façon suivante: a) ajout d'un point d'enquête en Kabylie orientale - la localité de Tighramt, située à $40 \mathrm{~km}$ à l'est de Chemini et b) ajout de huit points d'enquête en Kabylie occidentale (Grande Kabylie, chef-lieu: Tizi Ouzou). Ces huit points se répartissent sur le nord et le sud de la région de la façon suivante :

9 - au nord : trois localités

10 - Makouda : au nord-ouest de la région, à $20 \mathrm{~km}$ de la wilaya de Tizi Ouzou et à $20 \mathrm{~km}$ de la mer Méditerranée.

11 - Alma et Freha : à $30 \mathrm{~km}$ de Tizi Ouzou, séparées de $15 \mathrm{~km}$ et situées à $45 \mathrm{~km}$ à l'est de Makouda.

12 - au sud : cinq localités 
13 - Berkouka et Souk El Thenine : au sud-ouest de la région, à $35 \mathrm{~km}$ et $22 \mathrm{~km}$ de Tizi Ouzou, respectivement, et séparées de $16 \mathrm{~km}$.

14 - Agouni Gueghrane et Aït Abdelouahab : à $40 \mathrm{~km}$ de Tizi Ouzou, séparées de $10 \mathrm{~km}$, et situées à $25 \mathrm{~km}$ à l'est de Berkouka et Souk El Thenine.

15 - Aïn El Hammam : à $50 \mathrm{~km}$ de Tizi Ouzou et à $35 \mathrm{~km}$ au nord d'Agouni Gueghrane et Aït Abdelouahab.

16 Au total, nous disposons donc d'un réseau de 10 points d'enquête et nous renverrons par la suite à ces points par les abréviations suivantes :

(5) Kabylie occidentale (Grande Kabylie, chef-lieu : Tizi Ouzou)

$\begin{array}{lll}\text { nord } & \text { Mk } & \text { Makouda } \\ & \text { A } & \text { Alma } \\ & \text { F } & \text { Freha } \\ \text { sud } & \text { B } & \text { Berkouka/Maatka } \\ & \text { SET } & \text { Souk El Thenine } \\ & \text { AA } & \text { Ä̈t Abdelouahab (commune d'Aït Toudert) } \\ & \text { AG } & \text { Agouni Gueghran } \\ & \text { AEH } & \text { Ä̈n El Hammam }\end{array}$

\section{Kabylie orientale (Petite Kabylie, chef-lieu : Béjaïa) \\ Ch Chemini \\ T Tighramt}

Ces dix points sont représentés sur la carte ci-dessous, générée avec le logiciel Mapinfo:

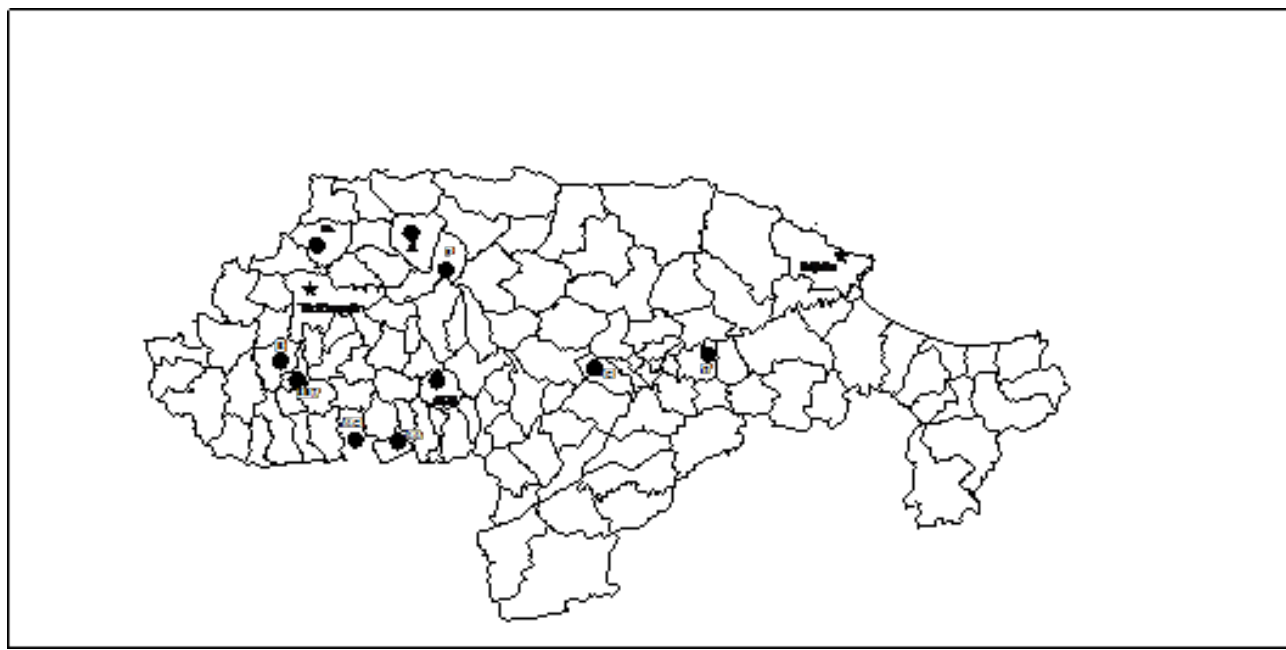

Nous avons présenté les phrases (en français) qui ont servi à l'élaboration du corpus de Bendjaballah \& Haiden $(2005,2013 a, b)$ aux locuteurs et nous leur avons demandé de les produire dans leur parler (date de l'enquête: février 2015). Afin d'assurer la compréhension des phrases proposées, nous avons choisi, pour chacun des points d'enquête sauf AEH, un(e) étudiant(e) de Master de l'université Mouloud Mammeri de Tizi Ouzou avec L1 kabyle, L2 arabe et L3 français. Ces étudiant(e)s, deux hommes et six femmes, sont âgés de 25 à 30 ans au moment de l'enquête. Ensuite, nous avons complété et vérifié les données obtenues pour les localités de Mk, B, SET et AG avec six locuteurs supplémentaires. A Mk et B, il s'agissait de quatre hommes, fonctionnaires, âgés de 40 à 45 ans, avec L1 kabyle, L2 arabe, L3 français et à SET et AG, de deux femmes au foyer, 
âgées de 30 et 40 ans, avec L1 kabyle, L2 arabe, L3 français. En ce qui concerne AEH enfin, l'un des auteurs de cet article [Samir Ben Si Saïd] est locuteur natif de ce parler; le questionnaire a également été soumis à un enseignant de français à la retraite, âgé de 61 ans.

\section{Un paradigme de complémenteurs complexes grammaticalisés : contraintes de taille et gabarit du constituant (III)}

Nous commençons par examiner la structure du complémenteur morphologiquement complexe (position (III)) et montrons que la taille des composants de ce complémenteur n'est pas libre mais obéit à des contraintes strictes. Nous en concluons que la préposition préfixée au complémenteur fait partie du gabarit du complémenteur, c'està-dire que le complexe P-C est grammaticalisé.

\subsection{L'identification du complémenteur et de l'opérateur interrogatif}

L'analyse requiert d'établir clairement l'identité du complémenteur $C$ et de l'opérateur interrogatif. Considérons à cette fin les exemples en (6)-(7). (6a-b) illustre des questions portant sur un PP dont la tête est $\mathrm{P}=s$ « avec », avec $\mathrm{C}=i$ et $a$ respectivement, (7a-b) illustre les configurations parallèles avec $\mathrm{P}=\mathrm{g}$ «dans». Dans chacun des cas, nous donnons deux exemples : dans le premier, l'opérateur interrogatif comporte l'élément pronominal $a f u$, dans le second, l'élément $a j .{ }^{4}$
a. $B$ anta

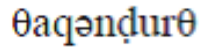
swaju
it $^{5} \quad$ iwala
$F$ anta
$\theta$ aqənd̦ur $\theta$
swaj
sit $^{5} \quad$ iwala
(I)
(II)
(III)
«Avec quelle robe l'a-t-il vue?»
b. $B$ anta

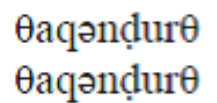
swaj
$\mathrm{at}^{5}$
iwali
$F$ anta
(II)
$\mathrm{sat}^{\mathrm{s}}$
iwali
(I)
(III)

«Avec quelle robe la verra-t-il?»

(7)
a. $A E H$
F
anwa
axxam
axxam
$\begin{array}{ll}\text { gg"aju } & \text { it }^{5} \\ \text { ggaj } & \text { jit }^{5}\end{array}$
ruzan
(I)
(II)
(III)
ruzan
«Dans quelle maison 1'ont-ils attendue?»
b. $A E H$ anwa axxam ggwafu at $^{5}$ razun $F$ anwa axxam ggaj jat ${ }^{5}$ razun
$\begin{array}{ll}\text { (I) } & \text { (II) }\end{array}$
«Dans quelle maison l'attendront-ils?» 
21 Etant donné que $\mathrm{C}$ varie avec l'aspect et que l'opérateur interrogatif varie avec la $\mathrm{P}$, les exemples en (6) et (7) permettent d'établir la segmentation suivante, en particulier l'analyse de $a / i$ comme complémenteurs $:^{5}$

$$
\begin{aligned}
& \text { a. }\left[\begin{array}{ll}
s w a f u & \mathrm{i} / \mathrm{a}-\mathrm{t}^{\mathrm{s}}
\end{array}\right]=\mathrm{s}-\mathrm{wa} \mathrm{ju} \quad \mathbf{i} / \mathbf{a}-\mathrm{t}^{\mathrm{s}} \\
& {\left[\begin{array}{ll}
g{ }^{w a} a & i / a-t^{s}
\end{array}\right]=g-w a \int u \quad \mathbf{i} / \mathbf{a}-t^{s}} \\
& \mathrm{P}-a f u . \mathrm{EC} \quad \mathrm{C}-\mathrm{OD}: 3 \mathrm{fs} \\
& \text { b. }\left[\begin{array}{ll}
s w a j & i / a-t^{s}
\end{array}\right]=s-w a j \quad s-i / a-t^{s} \\
& \text { [ggaj } \left.\mathrm{i} / \mathrm{a}-\mathrm{t}^{\mathrm{s}}\right]=\mathrm{g} \text {-waj } \mathrm{j}-\mathbf{i} / \mathbf{a}-\mathrm{t}^{\mathrm{s}} \\
& \text { P-aj.EC P-C-OD:3fs }
\end{aligned}
$$

\subsection{Contraintes gabaritiques}

Certaines P présentent une allomorphie de taille : selon leur contexte d'apparition, elles ont une réalisation légère (= une consonne) ou bien lourde ( $>$ une consonne). Dans le parler de Chemini, la $\mathrm{P}$ préfixée sur le $\mathrm{C}$ ne peut être que légère (Bendjaballah \& Haiden 2005, 2013a, b). Ainsi, pour la P « dans », dont les deux allomorphes sont ðəj et $g$, on a :

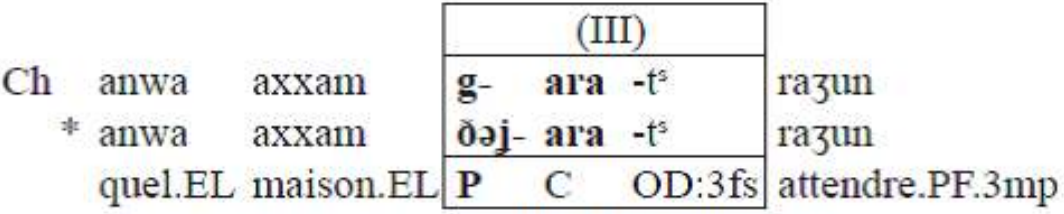

$$
\begin{aligned}
& \text { «Dans quelle maison vont-ils 1'attendre?» }
\end{aligned}
$$

23 L'élargissement du périmètre dialectal révèle que cette restriction doit être formulée de manière plus générale : il existe bien une contrainte de taille sur les éléments du complémenteur complexe $\mathrm{P}-\mathrm{C}$, mais celle-ci porte sur l'ensemble de la structure et non pas sur l'un ou l'autre de ses éléments. Les 10 parlers examinés illustrent en effet les 3 combinaisons suivantes : $\mathrm{P}$ réduite $-\mathrm{C}$ plein, $\mathrm{P}$ réduite $-\mathrm{C}$ réduit, $\mathrm{P}$ pleine $-\mathrm{C}$ réduit. $\mathrm{La}$ combinaison $P$ pleine $-C$ plein en revanche n'est pas attestée.

Nous illustrons cette généralisation par les $\mathrm{P}$ бəj « dans » et (a) et (b) respectivement ci-dessous) et le complementeur [+irrealis] ara, dont les deux allomorphes sont $a$ (léger) et ara (lourd). 
(10) $\underline{\text { P réduite }-\mathrm{C} \text { plein }}$

a. $\mathrm{P}=\partial \partial j ;$ allomorphe léger $=g(\mathrm{Ch}) / j(\mathrm{~A})$

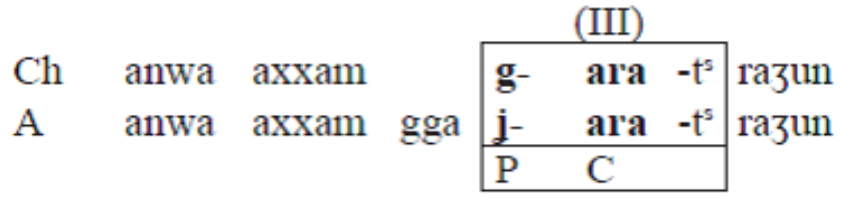

«Dans quelle maison l'attendront-ils?»

b. $\mathrm{P}=$ bəf ; allomorphe léger $=f(\mathrm{Ch}) / \boldsymbol{b}(\mathrm{AG})$

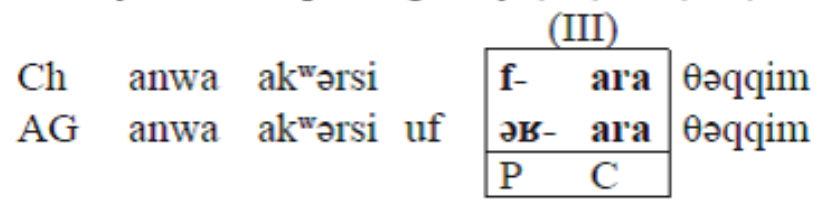

«Sur quelle chaise s'asseoira-t-elle?»

(11) $\quad \underline{\text { Préduite }-\mathrm{C} \text { réduit }}$

$\mathrm{C}=$ ara $;$ allomorphe léger $=a$

a. $\mathrm{P}=$ ठəj ; allomorphe léger $=j(\mathrm{~F}, \mathrm{Mk}, \mathrm{AEH}) / \partial(\mathrm{B}, \mathrm{SET})$

\begin{tabular}{|c|c|c|c|c|c|c|c|}
\hline & & & & \multicolumn{3}{|c|}{ (III) } & \multirow{5}{*}{$\begin{array}{l}\text { razun } \\
\text { razun } \\
\text { razun }\end{array}$} \\
\hline $\mathrm{F}, \mathrm{Mk}$ & anwa & axxam & ggaj & əј- & $\mathbf{a}$ & $-t^{5}$ & \\
\hline $\mathrm{B}, \mathrm{SET}$ & anwa & axxam & ggaj & әб- & a & $-t^{5}$ & \\
\hline $\mathrm{AEH}$ & anwa & axxam & i & $\mathbf{j}-$ & a & $-t^{5}$ & \\
\hline & & & & & C & & \\
\hline
\end{tabular}

«Dans quelle maison 1'attendront-ils?»

b. $\mathrm{P}=$ bəf ; allomorphe léger $=f(\mathrm{Mk}, \mathrm{AEH})$

\begin{tabular}{|c|c|c|c|}
\hline \multirow{3}{*}{$\begin{array}{l}\mathrm{Mk} \\
\mathrm{AEH}\end{array}$} & \multirow{4}{*}{$\begin{array}{lll}\text { anwa } & \mathrm{ak}^{\mathrm{w}} \text { ərsi } & \text { ffaji } \\
\text { anwa } & \mathrm{ak}^{\mathrm{w}} \text { ərsi } & \mathrm{i}\end{array}$} & \multicolumn{2}{|l|}{ (III) } \\
\hline & & f- a & өəqqim \\
\hline & & f- a & өəqqim \\
\hline & & $\mathrm{P} \quad \mathrm{C}$ & \\
\hline
\end{tabular}




$$
\underline{\mathrm{P} \text { pleine }-\mathrm{C} \text { réduit }} \mathrm{C}=\text { ara } \text { allomorphe léger }=a
$$

a. $\mathrm{P}=\partial \partial j$

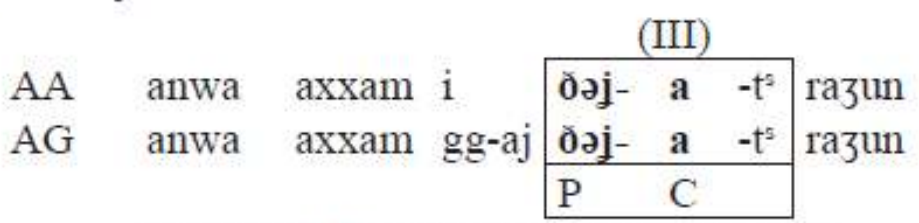

«Dans quelle maison 1'attendront-ils?»

b. $\mathrm{P}=$ bəf

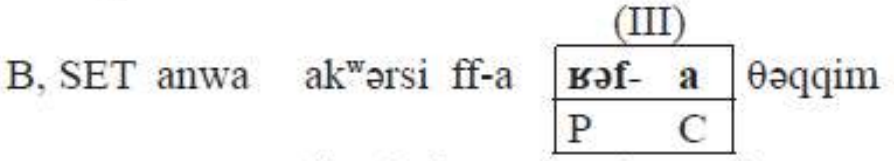

«Sur quelle chaise s'asseoira-t-elle?»

\subsection{Conclusion}

Si le complémenteur en position (III) est préfixé, une contrainte de taille sur la séquence $\mathrm{P}-\mathrm{C}$ s'applique dans tous les parlers: une préposition pleine n'est jamais suivie d'un complémenteur plein. Ceci indique que, dans tous les parlers examinés, la $P$ préfixale fait partie du gabarit du complémenteur. Nous en concluons que les séquences $\mathrm{P}-\mathrm{C}$ sont grammaticalisées comme complémenteurs prépositionnels interrogatifs. (Sur ce point, voir Chaker (1983) : 398, "Par leur stabilité et leur fréquence dans certains parlers, ces complexes sont certainement l'indice d'une tendance à la constitution d'un paradigme de supports spécifiques. »)

Une généralisation supplémentaire, mise en évidence dans notre corpus, vient confirmer cette conclusion. Dans certains cas, la P réalisée sur le C en (III) n'est pas identique à celle en (II). Ainsi, dans les exemples ci-dessous, la P en (II) est mais la $\mathrm{P}$ préfixée sur $\mathrm{C}$ en (III) est $j$, allomorphe léger de $\partial \partial j$ « dans ». ${ }^{6}$ Il est donc impossible d'analyser les structures de ce type comme impliquant un redoublement de la P. Le préfixe sur le $\mathrm{C}$ est grammaticalisé.

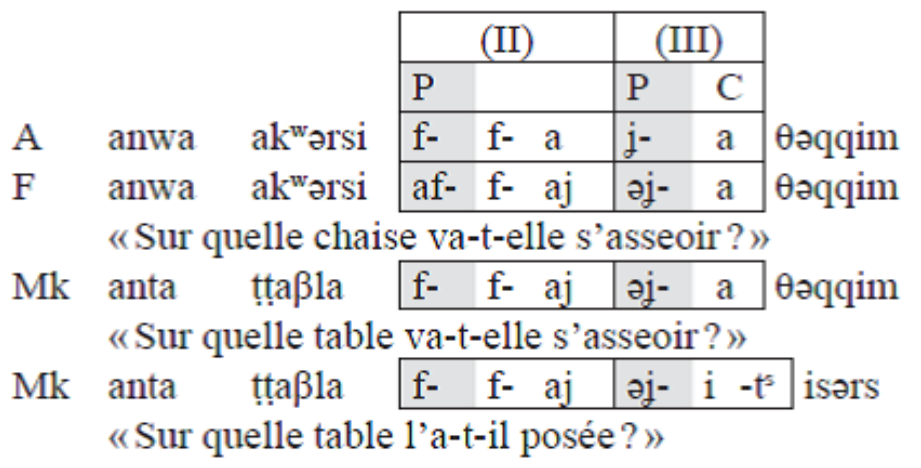




\section{La structure du constituant (II)}

Nous examinons maintenant la structure du constituant (II). Notre objectif est d'abord de mettre au jour l'identité des éléments de ce constituant puis de comprendre la façon dont constituant (II) et constituant (III) interagissent.

\subsection{Les positions de $P$, règles de distribution}

La configuration de départ est celle donnée en (1a) pour Chemini et rappelée ci-dessous en (14). Cette configuration est également celle attestée à Alma :

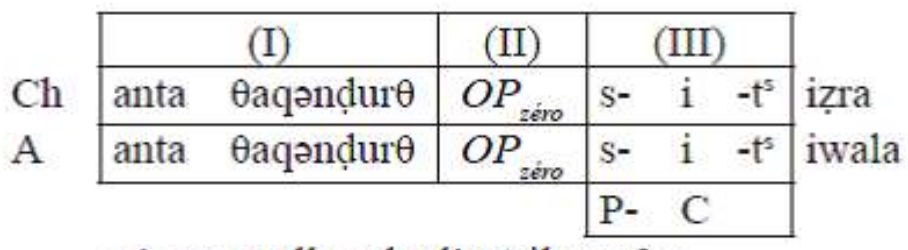

"Avec quelle robe l'a-t-il vue?»

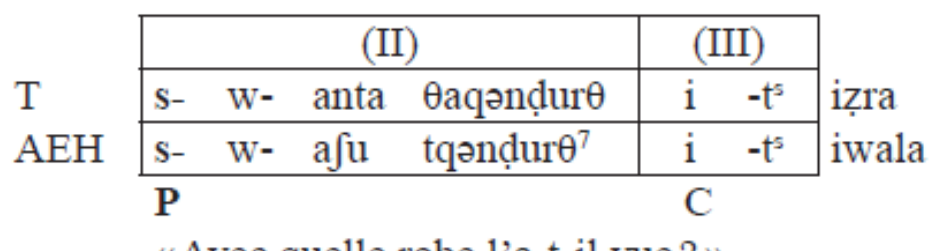

«Avec quelle robe 1'a-t-il vue?»

Cette structure comporte un constituant interrogatif sans $\mathrm{P}$ à la périphérie gauche (I) et un complémenteur complexe (III). Nous rappelons que le constituant anta $\theta$ aqəndur $\theta$ ne peut pas occuper la position (II) parce que sa forme syntaxique (syntagme nominal) n'est pas compatible avec la fonction qu'il doit remplir dans la phrase, celle de complément prépositionnel. Pour cette raison nous avons fait l'hypothèse d'un opérateur vide occupant la position (II).

Dans les 8 autres parlers que nous avons examinés, deux grandes familles de structures sont attestées :

a) des structures impliquant un constituant initial avec $\mathrm{P}$, mais pas de $\mathrm{P}$ sur le $\mathrm{C}$ en (III). Il s'agit de configurations comparables à (1c) : le constituant initial étant un syntagme prépositionnel, il peut réaliser sa fonction grammaticale dans la phrase sans l'intervention d'un opérateur vide. Pour cette raison, nous faisons l'hypothèse que ce constituant occupe la position (II). ${ }^{7}$

b) des structures impliquant trois constituants : (I) sans P, (III) avec ou sans P préfixée ${ }^{8}$ et, entre ces deux complexes, un constituant (II) de structure variable, grisé en (16) cidessous. En (16a), le constituant (II) comporte P et l'élément aj, P est préfixée sur C en (III). En (16b), (II) comporte P et l'élément afu, $\mathrm{P}$ n'est pas préfixée sur $\mathrm{C}$ en (III). Enfin en (16c), (II) ne comporte pas P mais soit $i / a j{ }^{9}$ soit $u$, P est préfixée sur $C$ en (III). 
(16)

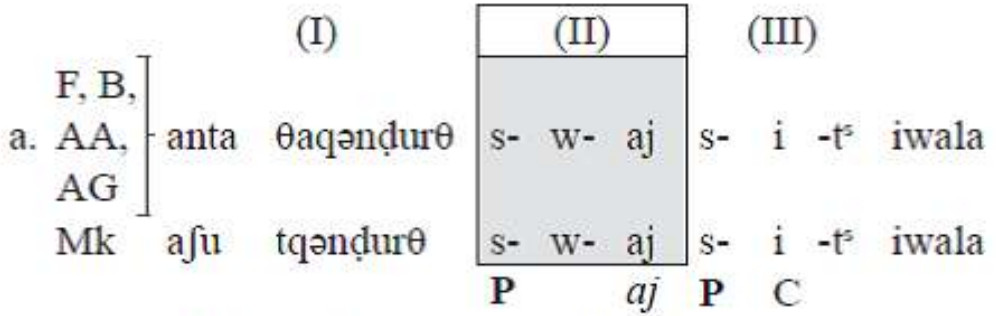

«Avec quelle robe l'a-t-il vue?»

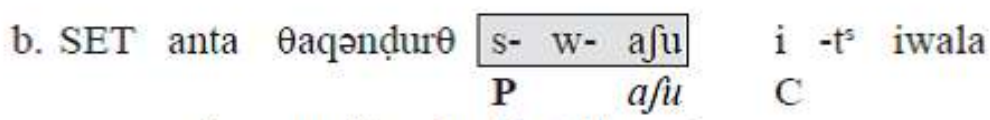

«Avec quelle robe l'a-t-il vue?»

\section{c. AEH anwa axxam i j- i $\quad-t^{5}$ ruzan aj/i $\mathbf{P} \quad \mathrm{C}$}

«Dans quelle maison l'ont-ils attendue?»

\begin{tabular}{rlll}
\hline $\mathrm{u}$ & rr- & $\mathrm{i}$ & oruћ \\
$\mathbf{P}$ & $\mathrm{C}$ &
\end{tabular}

«Vers quelle rivière est-elle allée?»

Examinons maintenant les 3 familles de structures attestées et que nous venons d'illustrer en (14), (15), (16). L'ensemble des structures relevées est résumé en (17): celles en (17a) impliquent un C nu, celles en (17b) un C préfixé.

Contraintes distributionnelles sur P

\begin{tabular}{|c|c|c|c|c|c|}
\hline & \multicolumn{3}{|c|}{ configuration attestée } & \multirow[t]{2}{*}{$e x$} & \multirow[t]{2}{*}{ parler } \\
\hline & (I) & (II) & (III) & & \\
\hline a.i & & $P-N P_{[+w h]}$ & $C$ & (15) & $\mathrm{T}, \mathrm{AEH}$ \\
\hline a.ii & $N P_{[+w h]}$ & $P-x$ & $C$ & $(16 b)$ & SET \\
\hline b.i & $N P_{[+w h]}$ & $P-y$ & $P-C$ & (16a) & $\mathrm{F}, \mathrm{B}, \mathrm{AA}, \mathrm{AG}, \mathrm{Mk}$ \\
\hline b.ii & $N P_{[+w h]}$ & $O P_{\text {zéro }}$ & $P-C$ & (14) & $\mathrm{A}, \mathrm{Ch}$ \\
\hline b.iii & $P-N P_{[+w h]}$ & $O P_{\text {zéro }}$ & $P-C$ & (1b) & $\mathrm{Ch}$ \\
\hline b.iv & $N P_{[+w h]}$ & $y$ & $P-C$ & $(16 c)$ & $\mathrm{AEH}, \mathrm{B}$ \\
\hline
\end{tabular}

où $x=$ constituant morphologiquement réalisé qui contient $a f u$

$y=$ constituant morphologiquement réalisé qui ne contient pas $a f u$

Un examen des contraintes de cooccurrence de $\mathrm{P}$ sur les 3 positions (I), (II) et (III) révèle que certaines combinaisons ne sont pas attestées. Il existe donc des règles de distribution de $\mathrm{P}$. Afin de comprendre ces règles, nous devons tout d'abord examiner en détail la nature des constituants symbolisés par $x$ et $y$ en (17), i.e. des éléments aj/i, afu et $u$.

\subsection{La structure de (II) : à propos de aj/i, a $\int u$ et $u$}

Outre $\boldsymbol{P}-N P_{[+w h]}$ et $O P_{z e ́ r o}$, on trouve 3 structures en (II) : $\boldsymbol{P}$ - $\boldsymbol{x}, \boldsymbol{P}$ - $y$ et $y$. Pour la clarté, nous répétons ci-dessous les exemples correspondant à chacune de ces configurations en 
ajoutant les constructions datives, qui ne nous intéressent pas directement dans le cadre de cet article, mais qui nous permettent de compléter le tableau de la distribution de $P$ et $a j / i$, afu et $u$.

$$
P-x(=17 \text { a.ii })
$$

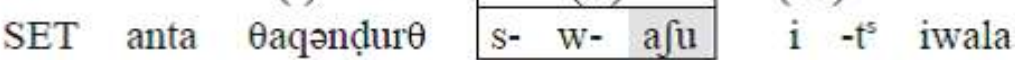
"Avec quelle robe 1'a-t-il vue?»

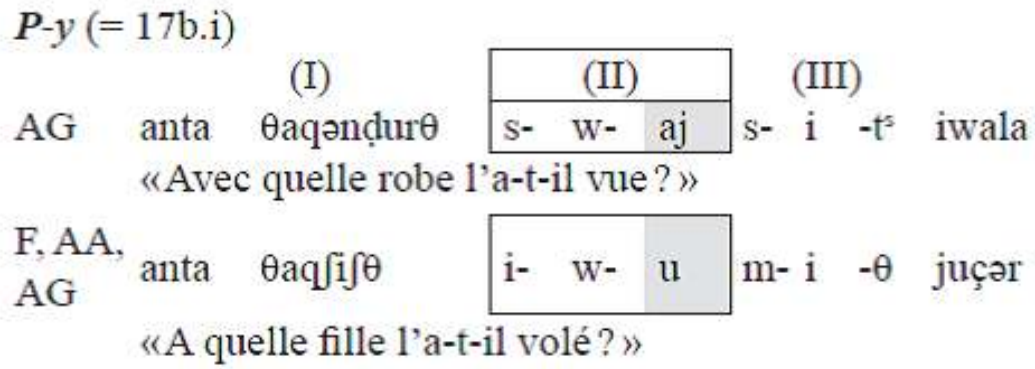

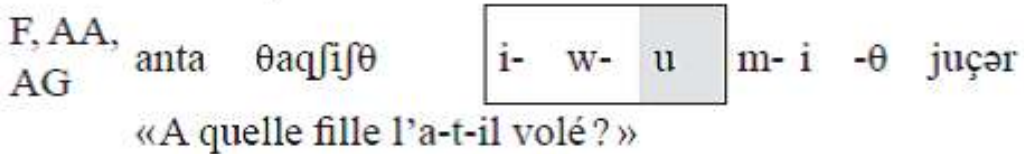

(20) $\quad y(=17 \mathrm{~b} . \mathrm{iv})$

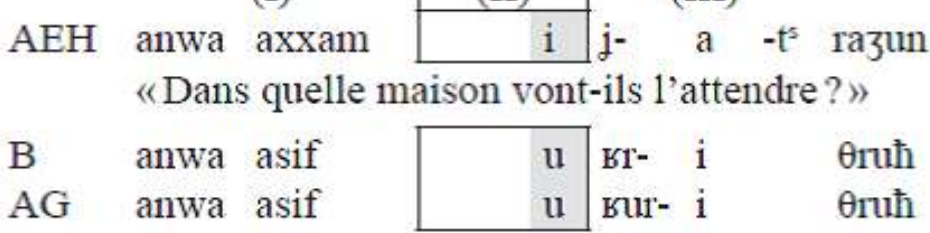

«Vers quelle rivière est-elle allée?»

Un examen précis des données révèle que $x=w a \int u$, i.e. afu à l'état construit (cas régi par la $\mathrm{P}$, marqué par $w$ - au masculin singulier). $y$ correspond à $a j / i$ ou $u$, à l'état construit (= waj, wu) si précédé de $\mathrm{P}$ et à l'état libre si non précédé de $\mathrm{P} .{ }^{10}$

Nous considérons que a $\int u$ et $a j / i$ sont des éléments pronominaux. ${ }^{11}$ Le cas de l'élément $u$ est plus complexe : nous trouvons en effet $u$ précédé de $P$ dans les constructions datives (19), mais non précédé de $\mathrm{P}$ dans les contextes de type (20).

Nous proposons que $u$ est la variante de aj/i en contexte directionnel $: u$ fait surface dans des contextes directionnels impliquant un mouvement vers un but, par ex. avec la P bur « vers» en (20) et la construction dative (19) peut être considérée comme un exemple particulier de construction directionnelle. Nous faisons l'hypothèse que $u$ est sous catégorisé comme complément soit de la $\mathrm{P}$ dative $i$, soit d'une $\mathrm{P}$ directionnelle vide. Nous illustrons ces deux cas en $(21 a, b)$ respectivement. 
(21)

a. $u$ à 1 'EC sous $\mathrm{P}$ dative

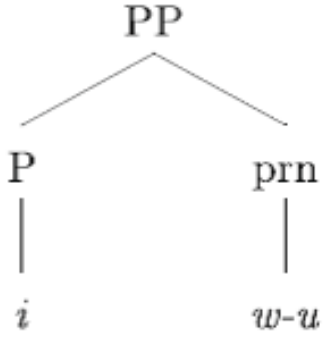

b. $u$ sous $\mathrm{P}$ vide

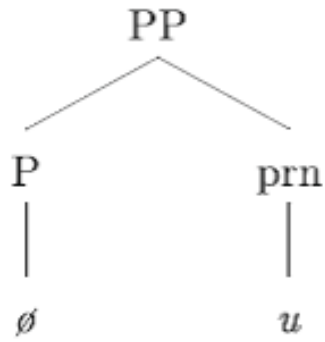

Enfin, le dernier cas est celui de AEH en (20). Dans cette configuration, on a, comme à B et $A G$, un seul élément sans $P$ en (II) mais cet élément est aj/i, et non $u$. Cette opposition $u$ vs aj/i à AEH s'étend aux constructions datives : là où $\mathrm{B}, \mathrm{F}, \mathrm{AA}, \mathrm{AG}$ ont $u(22 \mathrm{a}), \mathrm{AEH}$ a $a j / i(22 b)$.

a. B

(I)

b. $\mathrm{AEH}$ anta $\theta$ aqjif $\theta$ i $\mathrm{w}-\mathrm{aj}$ m- i $\quad-\theta$ juçər

anta $\theta a q f i f \theta$

\section{(II)} u $\mathrm{m}-\mathrm{i} \quad-\theta$ juçər i $\quad w-u \quad m-i \quad-\theta$ juçər "A quelle fille l'a-t-il volé?»

AEH emploie donc aj/i dans les constructions directionnelles. Nous interprétons ce fait de la façon suivante : la contrainte forçant la substitution de $a j / i$ par le morphème plus spécifique $u$ ne s'applique pas en AEH. La structure du domaine (II) de (20) à AEH est telle qu'en (23). Dans les autres parlers, cette structure est celle donnée précédemment en (21b).

(23)

\section{$a j / i$ sous $\mathrm{P}$ vide}

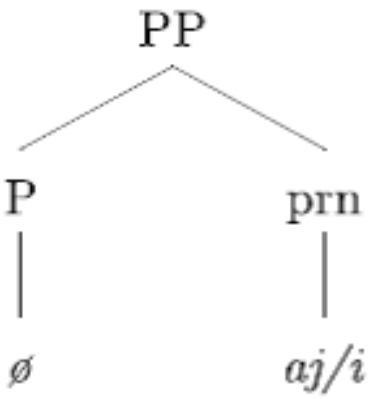

Pour résumer, le constituant (II) a la structure suivante :

(23)
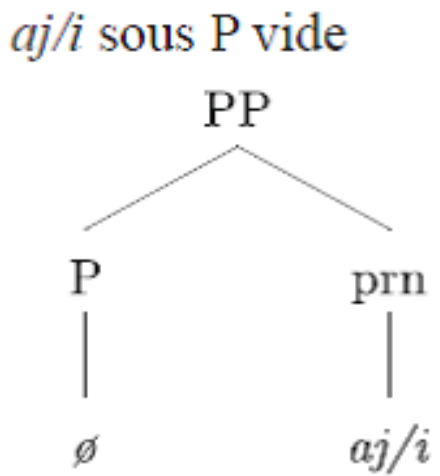
Nous examinons désormais les implications sur (III) de l'identification du complément de P par les pronoms aj/i et $u$ ou bien par afu.

\subsection{L'interaction entre (II) et (III)} (28, perfectif négatif).

(24) a. afu en (II) est incompatible avec P en (III).

b. aj/i et $u$ sont compatibles avec P en (III).

- le complémenteur nu (i.e. sans P préfixée) est précédé soit d'un PP interrogatif plein (17a.i), soit d'un constituant prépositionnel de type P-afu.EC (17a.ii).

- le complémenteur préfixé par $P$ est précédé soit d'un constituant prépositionnel de type $\mathbf{P}$-aj/i.EC (17b.i), soit d'un constituant prépositionnel de type $\mathbf{P}_{\text {zéro }}-\mathbf{u} / a j / i$ (17b.iv), soit enfin d'un opérateur zéro. ${ }^{12}$

Nous en déduisons la généralisation en (24):

b. aj/iet u sont compatibles avec $\mathrm{Pen}$ (III).

Cette généralisation émerge très clairement de l'ensemble de notre corpus, ainsi qu'illustré ci-dessous avec les complémenteurs $i(25,26$, perfectif), ara (27, futur) et ur

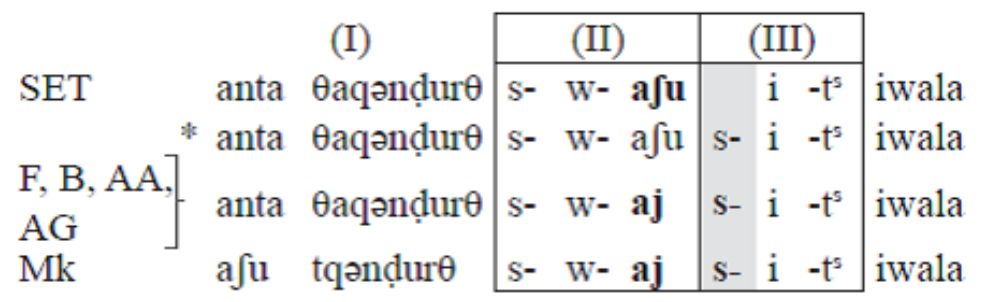

"Avec quelle robe l'a-t-il vue?"

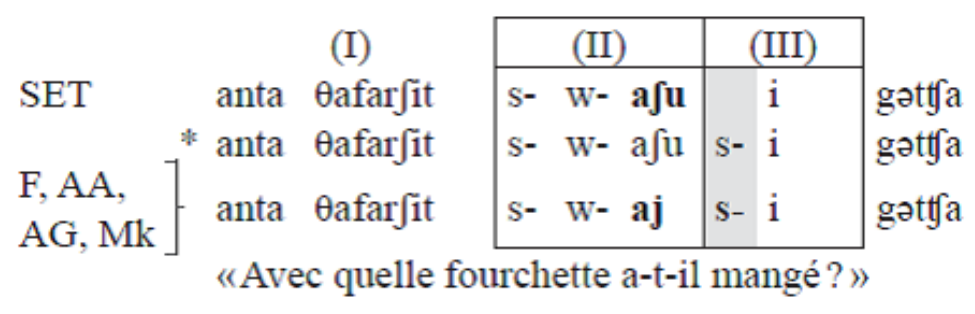

\begin{tabular}{|c|c|c|c|c|}
\hline \multirow{4}{*}{$\begin{array}{l}\mathrm{A}, \mathrm{SET} \\
\mathrm{F}, \mathrm{B}, \mathrm{AA}, \\
\mathrm{Mk} \\
\mathrm{AG}\end{array}$} & \multirow{2}{*}{$\begin{array}{l}\text { (I) } \\
\text { } \text { aaqənd̦ur } \theta\end{array}$} & (II) & (III) & \\
\hline & & $s-w-\mathbf{a} u$ & $a-t^{5}$ & iwali \\
\hline & anta $\theta$ aqəndur $\theta$ & s- w- aj & $s-a-t^{5}$ & iwali \\
\hline & aju tqənd̦ur $\theta$ & $s-w-\mathbf{a j}$ & s- $a-t^{5}$ & iwali \\
\hline
\end{tabular}

\begin{tabular}{|c|c|c|c|c|c|}
\hline \multirow{4}{*}{$\begin{array}{l}\text { SET } \\
\text { F, B, AA, } \\
\text { Mk } \\
\text { AG }\end{array}$} & & (I) & (II) & (III) & \multirow{4}{*}{$\begin{array}{l}\text { iwala ara } \\
\text { iwala ara } \\
\text { iwala ara }\end{array}$} \\
\hline & anta & $\theta$ aqənd̦ur $\theta$ & $s^{-}-w-a \int u$ & $u-t^{s}$ & \\
\hline & anta & சaqənd̦ur $\theta$ & $\mathrm{s}-\mathrm{w}-\mathbf{a j}$ & $S-u l-t^{s}$ & \\
\hline & $\begin{array}{l}\text { afu } \\
\text { «Av }\end{array}$ & tqəndur $\theta$ & $s-$ w- aj & s- $u-t^{5}$ & \\
\hline
\end{tabular}


aj et afu diffèrent à deux égards. Tout d'abord, $a \int u$ se comporte comme un nom en ce qui concerne la complémentation : il prend un complément au génitif (cf. AG en (27)). Par contraste, aj n'est jamais attesté dans cette configuration :

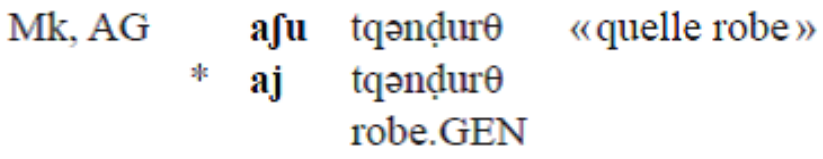

Ensuite, afu peut être employé comme pronom interrogatif en position (I), aj n'a jamais cette fonction :

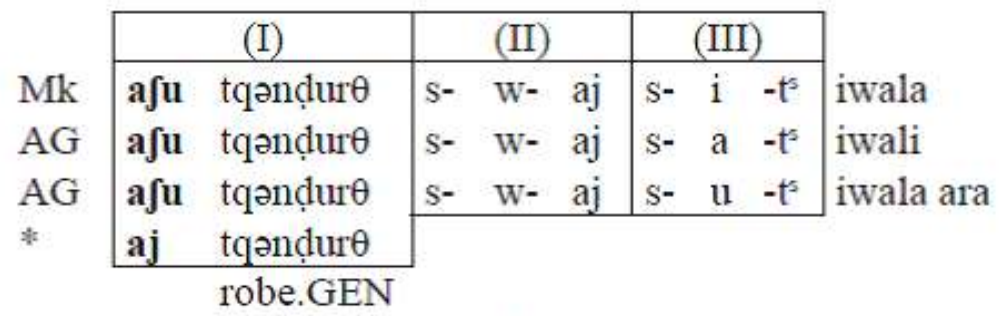

"Avec quelle robe l'a-t-il vue/la verra-t-il/ne l'a-t-il pas vue?»

Sur la base de ces contrastes, nous proposons que aj est un élément pronominal non interrogatif ([-wh]) tandis que afu est un élément pronominal interrogatif ([+wh]). Ainsi, $\mathrm{P}$-afu en position (II) est la manifestation d'un PP interrogatif plein (i.e. $P-N P_{[+w h]}$ ). Par conséquent, dans les constructions de type anta $\theta a q \partial n d u r \theta$ swafu it ${ }^{s}$ iwala « Avec quelle robe l'a-t-il vue? », la structure syntaxique des positions (II) et (III) est identique à celle des constructions bien connues dans lesquelles le PP interrogatif est en position (II), type swanta $\theta a q \partial$ dur $\theta$ it $i z$ izra.

\section{Conclusion}

Pour conclure, le tableau (17) peut-être précisé comme suit :

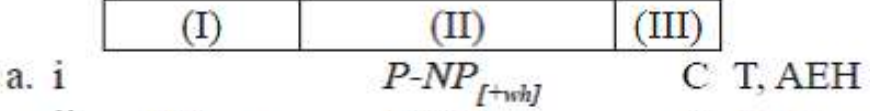

$$
\begin{aligned}
& \text { ii } N P_{[+w h]} \quad P-N P_{[+ \text {wh }]}^{[+ \text {C }]} \text { C SET } \\
& \text { b. i } \quad N P_{[+w h]} \quad P-N P_{[-w h]} \quad P-C \text { F, B, AA, AG, Mk } \\
& \text { ii } \quad N P_{[+w h]}^{[-w h]} \quad P_{z e ́ r o}-N P_{[-w h]}^{[-w h]} \quad P-C \text { AEH, B } \\
& \text { iii } N P_{[+ \text {wh }]} \quad P_{\text {zéro }}-N P_{[\text {-wh], zéro }} \quad P-C \mathrm{~A}, \mathrm{Ch} \\
& \text { iv } P-N P_{[+w h]} \quad N P_{[-w h], \text { zéro }} \quad P-C \text { Ch }
\end{aligned}
$$

Sur la base des configurations dans lesquelles (II) est morphologiquement réalisé (a.i-ii, b.i-ii), on constate que le complémenteur n'est préfixé par P que si (II) ne comporte pas de morphème interrogatif. Ceci nous permet d'extrapoler sur la valeur du trait [+/-wh] de l'opérateur zéro que nous avions admis dans les configurations (b.iii) et (b.iv). Puisque, dans ces configurations, $C$ est préfixé par $P$, il doit s'agir d'un opérateur [-wh]. Celui-ci est donc comparable à un pronom relatif plutôt qu'à un pronom interrogatif.

Les configurations (b.iii) et (b.iv) comportent toutes les deux un opérateur vide. Or les deux configurations se distinguent par la présence d'une P en position (I) en (b.iv) vs 
son absence en (b.iii). Nous supposons que cette différence en position (I) implique une asymétrie inverse en position (II) : absence de $\mathrm{P}$ en position (II) en (b.iv) vs sa présence en (b.iii).

\section{BIBLIOGRAPHY}

Bendjaballah S. \& M. Haiden. (2005). « The Grammar of Prepositions in Berber (Taqbaylit) », LingBuzz/000261.

-. (2013a). « La représentation syntaxique des prépositions faibles du berbère de Chemini », in J. Tseng (éd.) Prépositions et postpositions : approches typologiques et formelles. Paris : Hermès Lavoisier, 87-123.

-. (2013b). « The representational anomalies of floating markers : light prepositions in Taqbaylit of Chemini ", in T. Biberauer \& I. Roberts (éds) Challenges to Linearization. Berlin/New-York : Mouton de Gruyter, 331-375.

Chaker S. (1983). Un parler berbère d'Algérie (syntaxe). Aix-en-Provence : Publications de l'Université de Provence.

Dell F. \& M. Elmedlaoui. (1989). « Clitic Ordering, Morphology and Phonology in the Verbal Complex of Imdlawn Tashlhiyt Berber ", Langues Orientales, Anciennes, Philologie et Linguistique 2 : 165-194.

-. (1991). « Clitic Ordering, Morphology and Phonology in the Verbal Complex of Imdlawn Tashlhiyt Berber. Part II ", Langues Orientales, Anciennes, Philologie et Linguistique 3 : 77-104. Guerssel M. (1987). « The Status of the Lexical Category Preposition in Berber : Implications for the Nature of the Construct State », in K. Hale (éd.) Studies in Berber Syntax. Cambridge, Mass. : Center for Cognitive Science, MIT, 159-190.

Merchant J. (2002). « Swiping in Germanic », in J.-W. Zwart \& W. Abraham (éds) Studies in Comparative Germanic Syntax. Amsterdam : John Benjamins, 295-321.

Mettouchi A. (2005). Contribution à l'étude de la prédication. De la sémantique énonciative à la typologie. Mémoire d'HDR, Paris : INALCO.

Ouali H. (2011). Agreement, Pronominal Clitics and Negation in Tamazight Berber. London, New Delhi, New York, Sydney : Bloomsbury Publishing.

Ouhalla J. (2001). Clitics and edges : a comparative study of clitic placement and grammaticalization in Berber, ms. Queen Mary, University of London.

-. (2005). « Clitic-placement, grammaticalization and reanalysis in Berber », in G. Cinque \& R. Kayne (éds) The Oxford Handbook of Comparative Syntax. Oxford : Oxford University Press, 607-638.

Ouhalla J. \& A. El Hankari (2012 / ce volume). «Wh-clitic-doubling and wh-Cliticisation », Corpus 14. [Rééd. de Iberia vol. 4, http://dialnet.unirioja.es/ejemplar/327045. ISSN-e 1989-8525]

Penchoen T. (1973). Tamazight of the Ayt Ndhir. Los Angeles : Undena Press. 


\section{NOTES}

1. Abréviations : $\mathrm{EL}$ : état libre, EC : état construit, OD : objet direct, OI : objet indirect, $\mathrm{PF}$ : perfectif. Noter la présence du pronom enclitique $3 \mathrm{fs}-\mathrm{s}$ à la droite du complexe P-C. La distribution de ce pronom est régie par des contraintes indépendantes, $c f$. Dell \& Elmedlaoui $(1989,1991)$, Ouhalla $(2001,2005)$, Ouali (2011) entre autres.

2. Sur ce point, voir aussi les sections 3 et 4 de cet article.

3. Pour des données comparables dans d'autres langues berbères, voir Ouhalla \& El Hankari (2012/ce volume).

4. Nous revenons sur le statut de aj/afu plus bas en section 4.2.

5. On notera que la séquence [gg ${ }^{\mathrm{w}} \mathrm{a} \mathrm{Ju}$ ] correspond phonologiquement à /g-wa $\mathrm{fu} /$ et [ggaj] à /gwaj/. Ainsi, on doit poser la règle $\mathrm{g}+\mathrm{w} \rightarrow\left[\mathrm{gg}^{\mathrm{w}}\right]$ dans le premier cas et $\mathrm{g}+\mathrm{w} \rightarrow[\mathrm{gg}]$ dans le second cas. Nous retrouvons ici un phénomène bien connu: la réalisation de la labiovélarisation en général est sujette à une variation dialectale. Nous laissons la description précise de cette variation pour un travail ultérieur.

6. Notons que la généralisation précédente s'applique : la séquence $P$ - $C$ n'est jamais de type « $P$ pleine - $\mathrm{C}$ pleine». Par ailleurs, dans tous les parlers, la règle phonologique $/ \mathrm{f}+\mathrm{w} / \mathrm{-}$ [ff] s'applique : /f-waj/ -> [ffaj].

7. [tqənḍur $\theta$ ] est le nom $\theta a q \partial n d u r \theta ~ «$ robe » à l'EC précédé de la $\mathrm{P} n$ de génitif : sa forme sousjacente est $/ \mathrm{n}-\theta$ qəondur $\theta /$ et la règle $/ \mathrm{n}-\theta /$-> [t] s'applique. (II) doit ainsi être segmenté comme suit :

/s-waJun- $\theta$ qəndur $\theta /$ avec-chose[+wh].ECGEN-robe.EC

8. Nous revenons en détail sur ce point plus bas en section 4.3.

9. En (16c), $i$ est une réalisation de aj. A AEH, $i$ et aj sont en variation libre, avec néanmoins une tendance à employer aj dans le contexte phonologique $\mathrm{V}_{--}$. On a par ex. : anwa axxam $\mathbf{i}$ j-i- $\mathrm{t}^{\mathrm{s}}$ ruzan «Dans quelle maison l'ont-ils attendue ?» vs ð axxam-agi aj j-i- $\mathrm{t}^{\mathrm{s}}$ ruzan « C'est dans cette maison qu'ils l'ont attendue ». Dans la suite, nous noterons cet élément sous la forme aj/i.

10. Dans notre corpus, on trouve également $\mathrm{P}$ suivie de $w$-aji, mais sur un seul point d'enquête (à $\mathrm{Mk})$ et dans un seul exemple, le suivant :

Etant donné que cet élément n'apparaît que dans un exemple unique, nous le laissons de côté dans le cadre de cet article.

11. Pour une vision similaire, $c f$. Chaker (1983) : 398 : "combiné au nominal ay/i, le support prépositionnel acquiert des latitudes fonctionnelles similaires à celles des "relatifs" pronominaux » et pour une discussion, $c f$. Bendjaballah \& Haiden (2013a) : § 5.2.

12. Nous rappelons que l'existence de cet opérateur est motivée par l'incompatibilité de la catégorie DP avec la fonction de complément prépositionnel (17b.ii) et par le redoublement de la P (17b.iii). Dans les deux cas, une analyse simple de déplacement d'un PP (comme nous la proposons pour 17a.i) est intenable.

\section{ABSTRACTS}

The prepositional phrase in the left periphery in Taqbaylit

In this article we study the prepositional interrogative constructions of a set of 10 datapoints located in the Kabylie region in North-Eastern Algeria. Some of these constructions are characterized by the prefixation of the preposition $(\mathrm{P})$ to the complementizer $(\mathrm{C})$. We observe 
that $C$ shows up in its bare, non--prefixed form, if the specifier position of $C$ is realized by an overt interrogative phrase, If the specifier of $C$ is realized by a non--interrogative phrase, or if it is silent, then $\mathrm{C}$ carries a prepositional prefix. This article is part of a larger project on the phonology, morphology and syntax of prepositional phrases in the varieties of Taqbaylit Berber.

Dans cet article, nous examinons les constructions interrogatives portant sur un groupe prépositionnel dans un réseau de 10 points d'enquête en Kabylie. Certaines de ces constructions sont caractérisées par la préfixation de la préposition (P) sur le complémenteur (C). Nous mettons en évidence que si, dans une construction interrogative portant sur un complément prépositionnel, $C$ est spécifié par un constituant interrogatif, alors $C$ ne porte pas de $\mathrm{P}$ préfixée. Si $C$ est spécifié par un constituant non interrogatif ou bien si le spécificateur de $C$ n'est pas réalisé morphologiquement, alors $\mathrm{C}$ est préfixé par $\mathrm{P}$. Cet article fait partie d'un projet plus large portant sur la phonologie, la morphologie et la syntaxe des groupes prépositionnels dans différentes variétés du taqbaylit.

INDEX

Keywords: prepositional phrase, interrogation, left periphery, Taqbaylit Berber, microvariation Mots-clés: groupe prépositionnel, interrogation, périphérie gauche, taqbaylit, microvariation

\section{AUTHORS}

SABRINA BENDJABALLAH

UMR 6310 LLING - CNRS \& Université de Nantes

\section{SAMIR BEN SI SAIID}

UMR7320 BCL CNRS

\section{MARTIN HAIDEN}

Université Lille 3 \& CNRS UMR 8163 STL 